\title{
Development of Step Cooling Control System after Rolling Based on Automatic Feedback Control in Hot Strip Mill
}

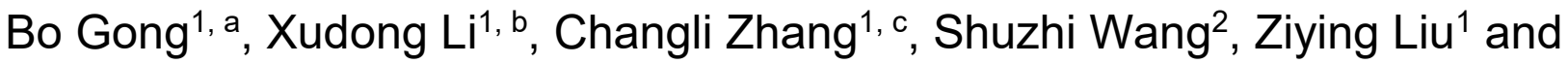 \\ Fengqin Wang ${ }^{1}$
}

1Shougang Research Institute of Technology, Beijing, 100043, China;

2Shougang Qian'an Iron \& Steel Company, Qian ‘an, 064400, China.

agongbo@shougang.com.cn, blixudong@shougang.com.cn, ${ }^{\mathrm{c}}$ zhangchangli@shougang,com.cn

Keywords: Hot strip mill, Cooling control, Step cooling, Feedback control, Mathematical model.

\begin{abstract}
Combined with a hot strip mill line, the laminar and step cooling mathematical models for cooling after rolling were introduced. The mathematical models mainly include air cooling model, water cooling model, setup control model, feed forward control model and feedback control model. Due to the delay of the feedback control, the original system could not achieve high precision control of the coiling temperature. Based on the process requirements of rolling dual phase steel, a new step cooling control system based on SMITH automatic feedback control was developed, and the cooling path and the configuration of the spray were re-divided. The experimental application showed that the optimized system had a stable operation and high control precision, the control precision of MT and CT is about $95 \%$, which had significantly improved the performance of the product and laid a foundation for the rolling of new steels.
\end{abstract}

\section{Introduction}

In order to ensure the processing and mechanical properties of strip steel, Laminar cooling control after strip rolling become an important part of hot rolling process. Laminar cooling control process control the strip coiling temperature by cooling water and strip speed. In recent years, with the development of society and the progress of science and technology, the demand for dual phase steel, duplex steel and high strength steel keep growing $[1,2,3,4]$. According to the technological requirements of these steel grades, the traditional cooling methods such as front cooling and post cooling can not meet the performance of their products, therefore [5], the step cooling process is applied to the cooling after rolling of these steels. Step cooling uses the cooling paths of water cooling, air cooling and water cooling to ensure duplex or multi-phase structure precipitation and achieve special organization performance requirements.

\section{Control Strategy and Mathematical Model of Laminar Cooling}

\subsection{Laminar Cooling Equipment.}

The laminar cooling device is generally divided into the main cold area and the fine cooling zone, located between the finishing mill and the coiler. This equipment contains 22 sets of collecting pipes, the first 20 groups are mainly cold area, each group has 4 double U-type sprays, and the latter two groups are the fine cooling zone, each group has 8 single U-type sprays.

For the step cooling process, the first 10 sets of collecting tubes are FFE as front section of the cooling zone, and the 11 to 18 sets are FFL as the rear section cooling zone, and the 19-20 set of collecting tube regions is the CTDC as velocity dynamic correction zone, and the 21-22 set of collecting tube regions is the FBK as fine tuning feedback zone.

\subsection{Mathematical Model of Laminar Cooling.}

The step cooling zone is mainly composed of two water cooling zones and a section of air cooling zone $[6,7]$.

The mathematical model of water cooling temperature drop is: 


$$
T=\frac{1000 * L w * Q}{3600 * v^{*} C_{p}^{*} \rho^{* h}}
$$

The mathematical model of air cooling temperature drop is:

$$
T_{a}=\left(\frac{6 * \varepsilon * \sigma}{\rho^{*} c_{p} / 1000} * \Delta \tau+\frac{1}{\left(t_{f}+273\right)^{3}}\right)^{-\frac{1}{3}}-273
$$

In the form, $T$ means the temperature drop in water cooling zone, ${ }^{\circ} \mathrm{C} ; L w$ means the length of water cooling zone, $\mathrm{m} ; \mathrm{Q}$ means the total heat flux density of upper and lower collecting pipes in water cooled zone, $\mathrm{kHz} /(\mathrm{m} 2 \cdot \mathrm{h}) ; v$ means the speed of strip steel, $\mathrm{m} / \mathrm{s} ; C_{p}$ means the specific heat capacity, $\mathrm{kHz} /\left(\mathrm{kg} \cdot{ }^{\circ} \mathrm{C}\right) ; \rho$ means the density of strip steel, $\mathrm{kHz} / \mathrm{m} 3 ; h$ means the thickness of step steel, $\mathrm{m} ; T_{a}$ means the temperature drop in air cooling zone, ${ }^{\circ} \mathrm{C} ; \varepsilon$ means thermal radiation coefficient of strip; $\sigma$ means Boltzmann's constant, $\mathrm{W} /(\mathrm{m} 2 \cdot \mathrm{K} 4) ; t_{f}$ means the target temperature of $\mathrm{FM}$ delivery, ${ }^{\circ} \mathrm{C}$.

The length of water cooling zone $L w$ is determined by the cooling rate and the target temperature. For example, in the setting stage, the front section of the water cooling zone generally adopts the rapid cooling in the front section. The cooling water flow required for the target middle point temperature MT is reached according to the finishing temperature FDT of the finishing rolling, and then the collection pipe is allocated according to the cooling rate S1 of the water cooling zone in the front section of the double section cooling process. [8] The calculation process of $L w$ is as follows:

$$
\begin{array}{r}
t_{1}=\frac{T_{F D}-T_{M}}{S_{1}} \\
L_{w}=v^{*} t_{1}
\end{array}
$$

In the form, $t_{1}$ means the cooling time of first water cooling zone, $\mathrm{s} ; T_{F D}$ means the measured temperature of FM delivery, ${ }^{\circ} \mathrm{C} ; T_{M}$ means the target temperature of middle point, ${ }^{\circ} \mathrm{C} ;{ }^{S_{1}}$ means the cooling rate of first water cooling zone, ${ }^{\circ} \mathrm{C} / \mathrm{s} ;, L w$ means the length of water cooling zone,m; $v$ means the speed of strip steel, $\mathrm{m} / \mathrm{s}$.

\section{Step Cooling Based on Automatic Feedback Control}

The cooling control models after rolling generally include setup calculation, feedforward calculation, feedback calculation and self-learning calculation. In step cooling process, it mainly includes the setup calculation of the two-stage cooling zone, the feedforward calculation of the twocooling zone, and the self-learning calculation of the two target temperatures. The setup calculation of step cooling is shown in the following figure:

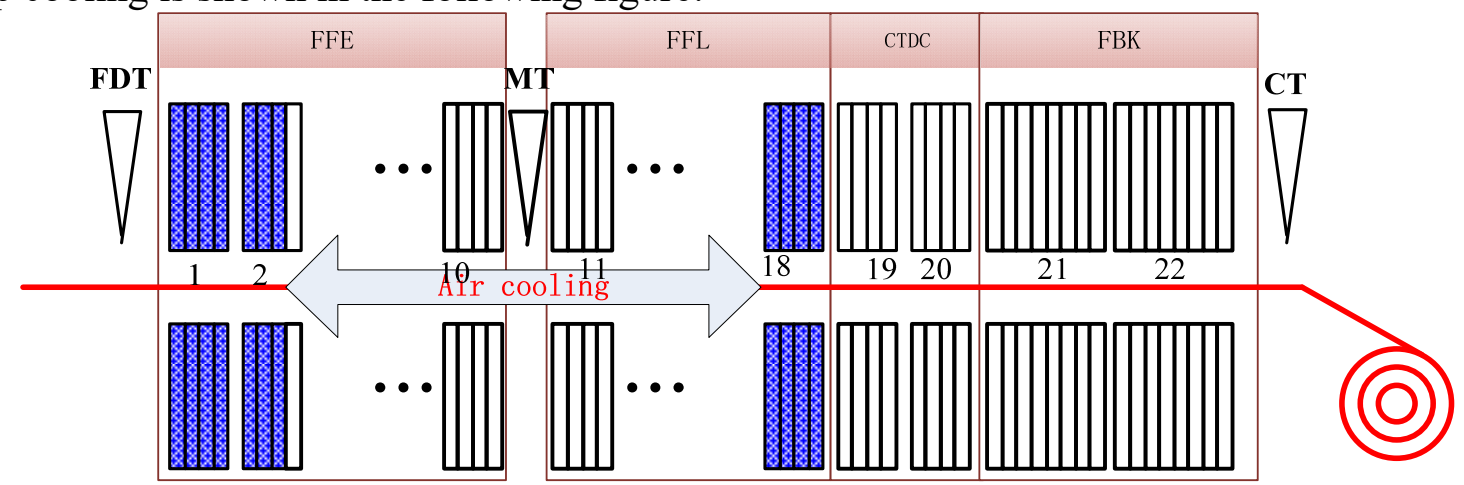

Fig 1. Original setup of step cooling.

Due to the special cooling path of the step cooling process, the general dual section cooling model has no real feedback module but is superimposed on the actual detection error of the pyrometer to the feedforward module. Due to the long-distance characteristic of laminar cooling, large delay control appears. In order to solve the delay, we redistributed the cooling zone, combined the dynamic velocity 
correction zone, the fine tune feedback area and the rear main cooling zone into second main cooling zones, and also responsible for the feedforward calculation and feedback calculation of the second section cooling zone. In the fine-tuning feedback area, the Smith feedback controller is used to perform the coiling temperature feedback calculation. The new setup calculation of step cooling is shown in the following figure:

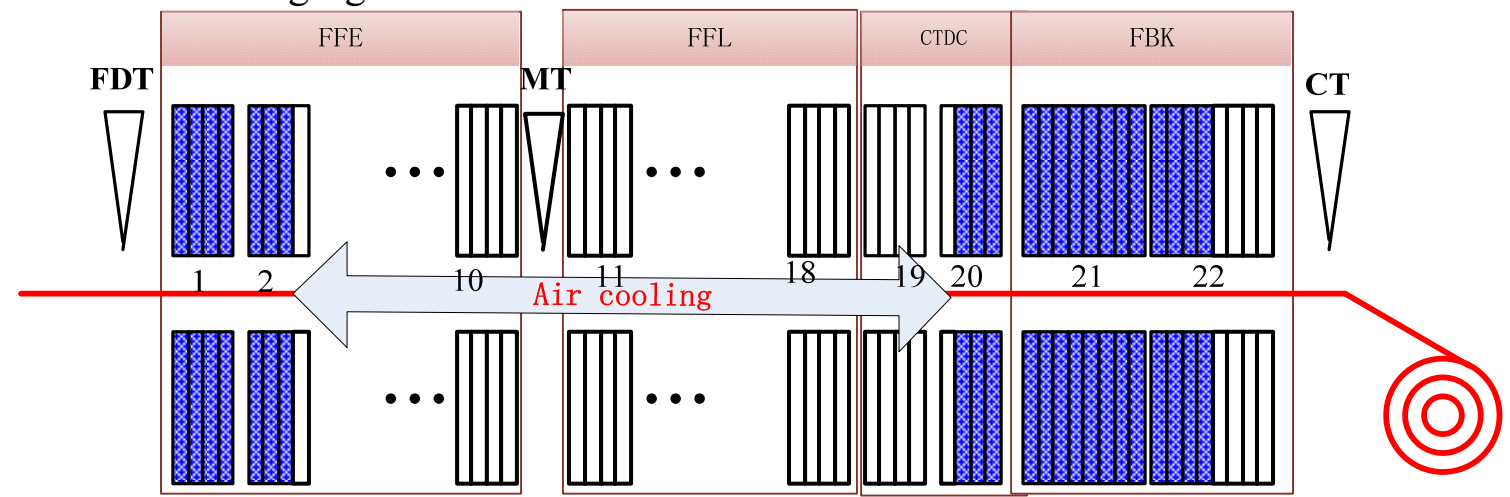

Fig 2. New setup of step cooling.

First, Model calculate the temperature drop of second sections of cooling zone according to the formula (1) and (2), and then calculate the length of the second section cooling zone according to the cold speed requirement of the main cooling zone and the formula (3) and (4), and then distribute the pipe configuration evenly in the second section cooling zone according to the principle of collecting tube distribution [9].

When the strip head reaches FDT and MT, the set tube configuration of the first cooling zone FFE and the second section cooling zone FFL is dynamically adjusted according to the measured temperature [10]. This is the feedforward calculation control of FFE and FFL, the control strategy is as follows:

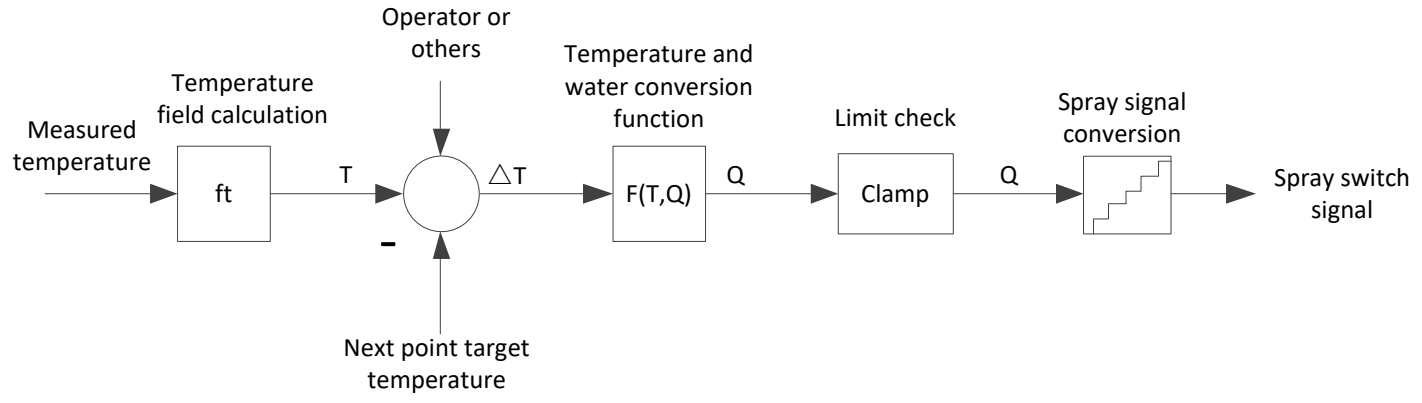

Fig 3. Feed forward control model.

When the strip reaches CT, according to the error between the measured temperature and the target temperature, the SMITH feedback controller is used to dynamically adjust the spray configuration of the FBK in the fine-tuning feedback zone. This is the automatic feedback control of the coiling temperature in the dual step cooling process.

$$
\begin{gathered}
\Delta T_{E}=T_{C T}^{d y-A I M}-T_{C T}^{A C T}-Y_{M 1}+Y_{M 22} \\
\Delta T_{C}=\text { smt_gain } *\left\{\begin{array}{c}
\left.\Delta T_{E}-\Delta T_{E}^{U B} \cdots \cdots \Delta T_{E}\right\rangle \Delta T_{E}^{U B} \\
\Delta T_{E}-\Delta T_{E}^{L B} \cdots \cdots \Delta T_{E}<\Delta T_{E}^{L B} \\
0 \cdots \cdots \cdots \text { otherwise }
\end{array}\right.
\end{gathered}
$$

In the form, $T_{C T}^{d y-A I M}$ means dynamic target coiling temperature, ${ }^{\circ} \mathrm{C} ; T_{C T}^{A C T}$ means measured coiling temperature, ${ }^{\circ} \mathrm{C} ; Y_{M 1}$ means the pre-calculation temperature of the sample section at the entrance of the fine tuning zone at the position of the coiling pyrometer, ${ }^{\circ} \mathrm{C} ; Y_{M 22}$ means the pre-calculation temperature for the sampling section of the pyrometer location at the inlet of the fine adjustment zone, ${ }^{\circ} \mathrm{C} ; \Delta T_{E}^{U B}$ and $\Delta T_{E}^{L B}$ means the limit check, ${ }^{\circ} \mathrm{C}$; smt_gain means the gain coefficient; $\Delta T_{E}$ and $\Delta T_{C}$ means the error of the SMITH feedback controller, ${ }^{\circ} \mathrm{C}$. 


\section{Application and Effect}

After the automatic feedback control is put into the control, the control strategy of the coiling temperature is increased by the double section cooling process, which solves the problem of the feedback delay of the original control strategy and greatly improves the control precision of the coiling temperature. Figure 4 is the MT and CT temperature curve before and after the automatic feedback use. It can be seen from the diagram that, after the input of the automatic feedback control, the real-time adjustment of the collection tube configuration in the fine-tuning feedback area makes the temperature control of the head and tail of the CT more smooth and accurate, and the full-length temperature is well controlled.

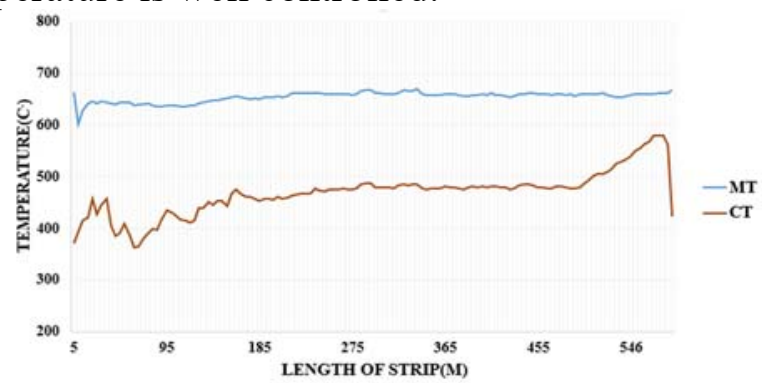

(a)Before use automatic feedback

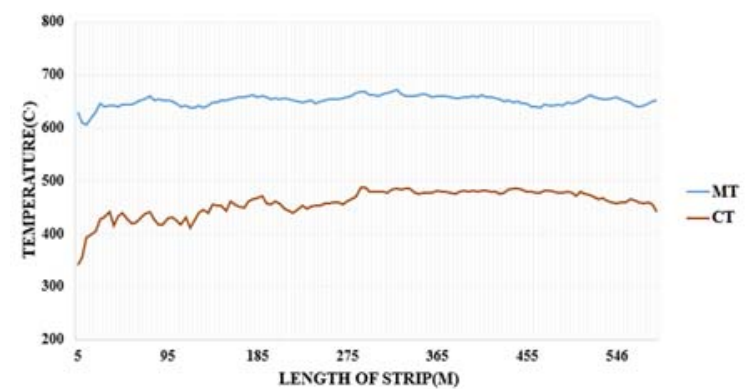

(b) after use automatic feedback

Fig 4. MT and CT curves with the automatic feedback control.

With the automatic feedback control, the system runs stably and reliably. At present, this process is used in double phase steel, complex phase steel, part of high strength container and beam steel. The temperature of the rolling process, MT and CT control precision is high, and the temperature and performance uniformity in the direction of strip length are ensured. Table 1 is the statistics of the hit rate of each type of steel.

Table 1. Statistics of the hit rate of each type of steel.

\begin{tabular}{ccccc}
\hline \hline steel name & Target MT & MT PctOn & Target CT & CT PctOn \\
\hline CPW800-P & $530^{\circ} \mathrm{C} \sim 590^{\circ} \mathrm{C}$ & $95.04 \%$ & $490^{\circ} \mathrm{C} \sim 550^{\circ} \mathrm{C}$ & $94.14 \%$ \\
FB60-P & $630^{\circ} \mathrm{C} \sim 690^{\circ} \mathrm{C}$ & $93.44 \%$ & $400^{\circ} \mathrm{C} \sim 500^{\circ} \mathrm{C}$ & $92.40 \%$ \\
HR580DP & $620^{\circ} \mathrm{C} \sim 680^{\circ} \mathrm{C}$ & $88.10 \%$ & $<=1500^{\circ} \mathrm{C}$ & $45.40 \%$ \\
SQ700J & $620^{\circ} \mathrm{C} \sim 680^{\circ} \mathrm{C}$ & $93.30 \%$ & $550^{\circ} \mathrm{C} \sim 620^{\circ} \mathrm{C}$ & $97.35 \%$ \\
S700L & $620^{\circ} \mathrm{C} \sim 720^{\circ} \mathrm{C}$ & $96.20 \%$ & $560^{\circ} \mathrm{C} \sim 650^{\circ} \mathrm{C}$ & $94.45 \%$ \\
\hline \hline
\end{tabular}

\section{Summary}

The main control strategies and mathematical models of step cooling are introduced according to the laminar cooling equipment. A new step cooling process based on automatic feedback control is proposed according to the requirement of microstructure and properties of dual phase steel and other steel grades. The experimental application shows that the dual section cooling process based on automatic feedback control can effectively solve the problem of the traditional step cooling feedback adjustment delay. The new process can accurately control the temperature of the middle point and the coiling temperature. The system operates reliably and has high temperature control precision for different steel types, the control precision of MT and CT is about $95 \%$, which had significantly improved the performance of the product and laid a foundation for the rolling of new steels.

\section{References}

[1]. Liu Enyang, Peng Liangui, Yuan Guo, Wang Zhaodong, Zhang Dianhua, Wang Guodong.Advanced run-out table cooling technology based on ultra fast cooling and laminar cooling in hot strip mill[J]. Journal of Central South University,2012,19(05):1341-1345.

[2]. FENG HuiJun, CHEN LinGen, LIU Xiong, XIE ZhiHui, SUN FengRui. Generalized constructal optimization of strip laminar cooling process based on entransy theory[J]. Science China (Technological Sciences),2016,59(11):1687-1695. 
[3]. Yuan Guo, Li Zhenlei, Li Haijun, Wang Zhaodong, Wang Guodong.Control and application of cooling path after rolling for hot strip based on ultra-fast cooling $[\mathrm{J}]$. Journal of Central South University,2013,20(07):1805-1811.

[4]. Opti mization and Model of Laminar Cooling Control Systemfor Hot Strip Mills[J]. Journal of Iron and Steel Research (International),2006(01):18-22.

[5]. XU Xiao-qing, HAO Xiao-dong, FU Song-lin, ZHOU Shi-guang, LIU Chang-sheng, ZHANG Qi-fu. Layer Flow Cooling Path Control Based on Temperature Observer[J]. Journal of Iron and Steel Research,2017,29(01):81-86.

[6]. Zhang Dianhua, Liu Enyang, Liu Weidong, Liu Xiaofeng. Optimization of Speed Feedforward Compensation for Hot Strip Rolling Cooling System[J]. Journal of Northeastern University (Natural Science),2011,32(04):516-519.

[7]. Opti mization and Model of Laminar Cooling Control Systemfor Hot Strip Mills[J]. Journal of Iron and Steel Research (International),2006(01):18-22.

[8]. Qing-yun SHA, Gui-yan LI, Li-feng QIAO, Ping-yuan YAN. Effect of Cooling Rate and Coiling Temperature on Precipitate in Ferrite of a Nb-V-Ti Microalloyed Strip Steel[J]. Journal of Iron and Steel Research International,2007,14(5).

[9]. Wang Lihui. Application Research on Control Method of Laminar Cooling Process of Hot Rolled Strip[J]. Shanxi Metallurgy,2017,40(03):87-88+90.

[10]. Sun Tiejun. High-precision prediction of strip coiling temperature and multi-objective optimization control strategy[D]. Beijing University of Science and Technology,2016. 